\title{
BMJ Open Quality Effectiveness of a submassive pulmonary embolism protocol to standardise patient evaluation and treatment
}

\author{
Kelly Galanos, ${ }^{1}$ Cassie Jaeger, ${ }^{2}$ Kevin Coakley, ${ }^{3}$ Peter White, ${ }^{4}$ David Griffen ${ }^{1}$
}

To cite: Galanos K, Jaeger C, Coakley K, et al. Effectiveness of a submassive pulmonary embolism protocol to standardise patient evaluation and treatment.BMJ Open Quality 2018;7:e000279. doi:10.1136/ bmjoq-2017-000279

- Additional material is published online only. To view please visit the journal online (http://dx.doi.org/10.1136/ bmjoq-2017-000279)

Received 5 December 2017 Revised 28 June 2018 Accepted 5 July 2018

\section{Check for updates}

(C) Author(s) (or their employer(s)) 2018. Re-use permitted under CC BY-NC. No commercial re-use. See rights and permissions. Published by BMJ.

${ }^{1}$ Division of Emergency Medicine, Department of Surgery, Southern Illinois University School of Medicine, Springfield, Illinois, USA ${ }^{2}$ Department of Operations Improvement, Memorial Health System, Springfield, Illinois, USA ${ }^{3}$ Department of Radiology, Southern Illinois University School of Medicine, Springfield, Illinois, USA

${ }^{4}$ Department of Internal Medicine, Southern Illinois University School of Medicine, Springfield, Illinois, USA

Correspondence to Dr Cassie Jaeger; Hawk.Cassie@mhsil.com

\section{ABSTRACT}

Treatment recommendations for submassive pulmonary embolus (SMPE), defined as pulmonary embolus (PE) resulting in right ventricular dysfunction and/or myocardial necrosis, vary. The objective of this study was to develop an investigative protocol at our tertiary care hospital to standardise the approach to patients with SMPE and to evaluate the effect of the protocol on process measures including consultation with cardiology and critical care physicians and time to echocardiogram and treatment. Triggered by right ventricle/left ventricle ratios $>0.9$, the protocol standardised ancillary studies and immediate consultation with critical care and cardiology. Postprotocol implementation, the percent of patients with SMPE evaluated by critical care specialists increased from $26 \%(19 / 74)$ to $93 \%(41 / 44)(p<0.001)$ and cardiology consultations increased from $35 \%$ (26/74) to $89 \%$ (39/44) $(p<0.001)$. Patient arrival to echocardiogram was reduced from 15 hours to 5 hours post-protocol implementation. In addition, average time to anticoagulation was reduced from greater than 7 hours to 3 hours 27 min post-protocol implementation. The protocol has helped to identify patients with SMPE and standardise the care they receive after diagnosis.

\section{PROBLEM}

Intensivists, emergency medicine physicians and cardiologists at a tertiary care centre in Illinois, USA, realised there was not a standardised definition or therapeutic approach to patients diagnosed with acute submassive pulmonary embolism (SMPE) at their facility. All patients diagnosed with SMPE were admitted to the hospital for further management; however, ancillary laboratory, diagnostic studies, patient placement (general floor vs intermediate care vs intensive care unit (ICU)) and treatment paths differed greatly despite similar patient presentations. It was noted in some cases that interventional cardiologists were consulted and catheter-directed thrombolysis was performed followed by ICU admission under the intensivists. In other cases, patients were admitted to the ICU for anticoagulation without cardiology input. Echocardiograms were not routinely obtained.
The objective of this project was to standardise the definition of SMPE, develop a protocol to streamline the approach to patients diagnosed with SMPE and encourage collaboration between specialties to develop treatment plans on a case-by-case basis using the appropriate objective data to guide their decisions.

\section{BACKGROUND}

In the USA, pulmonary embolism is listed as the cause of death in approximately 100000 cases annually. ${ }^{1}$ Risk stratification into low risk, submassive or massive pulmonary embolism (PE) categories should be initiated promptly to determine appropriate anticoagulant and thrombolytic therapy and reduce risk of recurrent thromboembolism and death. ${ }^{2-4}$

SMPE is defined as PE without hypotension with embolus resulting in right heart strain or myocardial necrosis. Depending on comorbidities or clot burden, the physical and clinical manifestations encompass a wide spectrum of disease. In addition to the variability in SMPE presentation, the risk stratification classifications vary between the American Heart Association, American College of Chest Physicians and European Society of Cardiology depending on recommended use of the simplified Pulmonary Embolism Severity Index score, right ventricle/left ventricle $(\mathrm{RV} / \mathrm{LV})$ ratio and elevated biomarkers. ${ }^{2-4}$

Due to variability in $\mathrm{PE}$ stratification and treatment modalities including ultrasound-enhanced thrombolysis, multidisciplinary consultation between specialties is advantageous to determining appropriate treatment. In 2016, Kabrhel et al, implemented a Pulmonary Embolism Response Team (PERT) composed of cardiology, cardiac surgery, echocardiography, emergency medicine, haematology, pulmonary/ critical care, radiology and vascular medicine. Once activated by a referring physician, the team reviewed clinical information in real 
time and returned treatment recommendations back to the referring provider. Implementation of the team was effective at coordinating multidisciplinary specialists to provide evidence-based care to patients with PE. ${ }^{56}$

\section{MEASUREMENT}

A retrospective cohort of 257 acute patients with $\mathrm{PE}$ was identified at our tertiary care centre from March 2015 to April 2016. In patients with undocumented RV/LV ratios (176/257), a radiologist measured ratios retrospectively. Exclusion criteria included diagnosis of PE prior to emergency department arrival or during hospital admission, $\mathrm{RV} / \mathrm{LV}$ ratio $\leq 0.9$, inability to measure $\mathrm{RV} / \mathrm{LV}$ ratio and diagnosis of PE per lung perfusion scan. Seventy-four retrospective patients met inclusion criteria. Consultation with critical care, consultation with cardiology, time to echocardiogram and time to anticoagulation treatment were analysed. Before the protocol was initiated, critical care was consulted for $26 \%(19 / 74)$ of patients and cardiology was consulted for $35 \%(26 / 74)$ of patients. Patient arrival to echocardiogram varied from 1 hour $35 \mathrm{~min}$ to greater than 2 days and patient arrival to anticoagulation varied from 59 min to greater than 2 days. The team believed the management approach for patients with SMPE should be standardised to reduce variability and improve patient care.

\section{DESIGN}

This project was reviewed by the local institutional review board. It was determined that this project was not research involving human subjects. From March 2015 to April 2016, a team of physician representatives from critical care, cardiology, radiology, vascular surgery and emergency medicine developed a SMPE definition and protocol to standardise the approach to patients with SMPE. Radiologists were instructed to document $\mathrm{RV} / \mathrm{LV}$ ratios as part of their CT interpretation. The SMPE protocol was triggered when an emergency medicine physician identified a patient with $\mathrm{PE}$ in addition to right heart strain on a CT scan, defined as a $\mathrm{RV} / \mathrm{LV}$ ratio $>0.9$. Once the protocol was activated, a cascade of dialogue and ancillary studies began including an official consultation with critical care and interventional cardiology and stat echocardiogram, which was pre-checked on the protocol's computerised order entry set. Emergency medicine, critical care and cardiology physicians conferred to determine the best course of action. Anticoagulation therapy was started immediately on diagnosis or suspicion (figure 1) (online Supplementary figures 1-3).

The protocol was activated in May 2016 and evaluated through April 2017. The overall goal of the project was to improve physician knowledge and comfort in recognising and treating patients with SMPE.

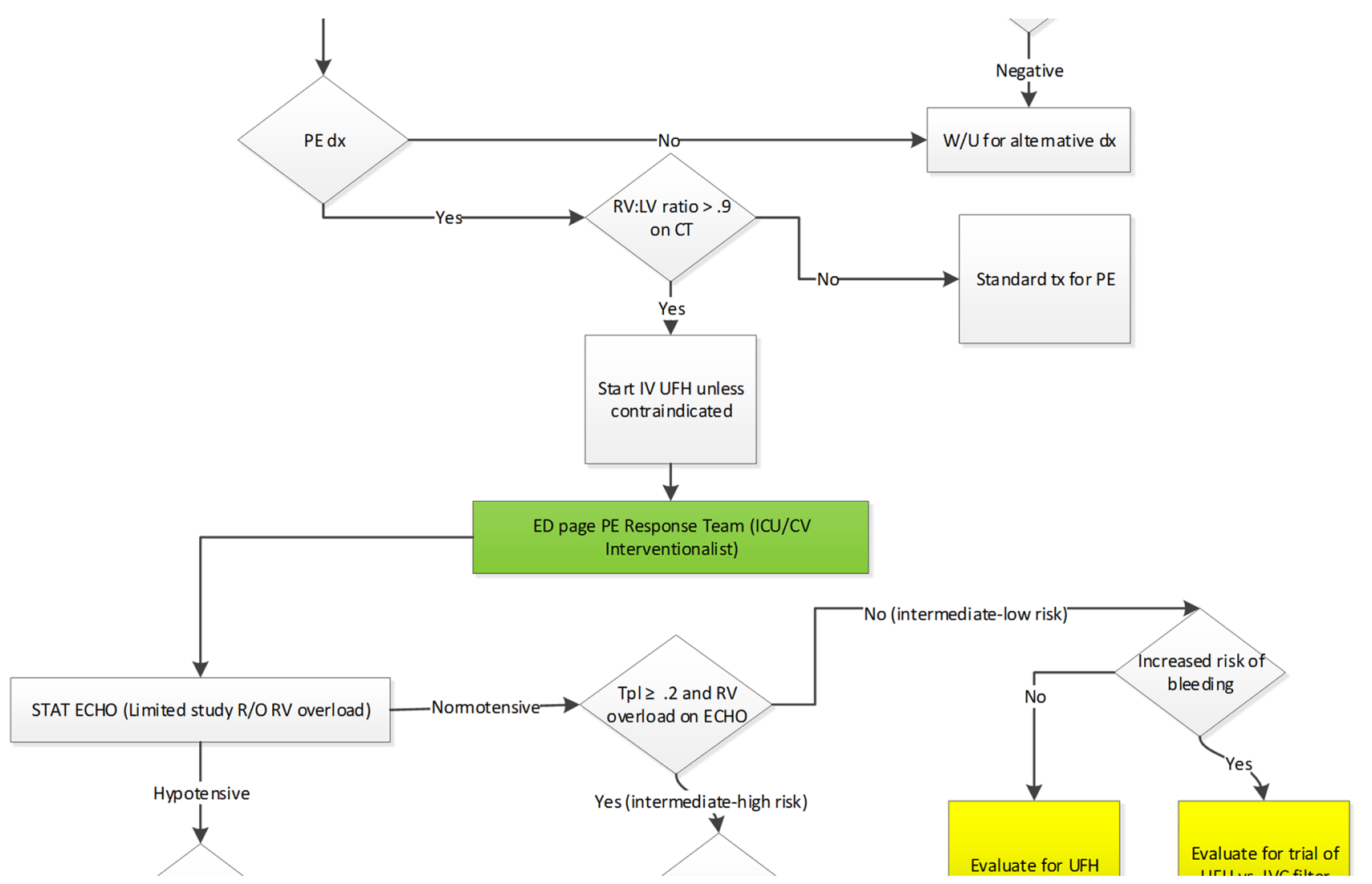

Figure 1 Flowchart of the submassive pulmonary embolus protocol. The protocol was triggered when a patient was identified with pulmonary embolism (PE) in addition to right heart strain, defined as a right ventricle/left ventricle (RV/LV) ratio $>0.9$. Once the protocol was activated, a cascade of dialogue and ancillary studies began including an official consultation with critical care and interventional cardiology and stat echocardiogram, which was pre-checked on the protocol's computerised order entry set. 


\section{STRATEGY}

Emergency medicine, critical care and cardiology leaders collaborated to define and standardise SMPE treatment. Before implementation of the protocol, staff from each specialty were educated on the importance of prompt SMPE recognition and initiation of therapy based on best practice guidelines. The SMPE protocol and process map were presented and discussed during hospitalist, critical care and emergency department physician meetings (online Supplementary figures 1-3). In addition, email and one-on-one conversations were used to reinforce use of the protocol. Project results and collaboration between emergency, critical care and cardiology physicians were discussed at the clinical performance meeting, department meetings and monthly project meetings with hospital leadership. To maintain sustainability, compliance with the protocol was monitored for each SMPE case by an emergency physician.

Differences in the percent of patients with SMPE with critical care or cardiology consultation were analysed by two-proportion test. Patient arrival to echocardiogram and patient arrival to anticoagulation therapy are displayed using Statistical Process Control charts in QI Macros in Excel. Data were analysed from May 2015 to April 2016 pre-intervention and May 2016 to April 2017 post-intervention. Two-proportion tests were performed in Minitab. A $p$ value of $<0.05$ was considered significant.

\section{RESULTS}

To assess the effectiveness of the protocol, 74 patients identified retrospectively were compared with 44 patients post-protocol implementation in May 2016. The SMPE protocol was not activated in $18 \%(8 / 44)$ of cases post-protocol implementation, even though the protocol was followed. The percent of patients with SMPE with a critical care consultation improved from 26\% (19/74) of patients to $93 \%(41 / 44)$ of patients post-protocol implementation $(\mathrm{p}<0.001)$ (figure 2A). Also, the number of patients with SMPE with cardiology consultations improved from $35 \%$
$(26 / 74)$ to $89 \%(39 / 44)$ after protocol implementation $(\mathrm{p}<0.001)$ (figure 2B).

Prior to protocol implementation, patient arrival to echocardiogram varied from 1 hour $35 \mathrm{~min}$ to greater than 2 days. Post-protocol implementation, average door to echocardiogram was reduced from 15 hours 2 min to 5 hours 1 min (figure 3 ). In addition, patient arrival to anticoagulation therapy was reduced from 7 hours $28 \mathrm{~min}$ to 3 hours $27 \mathrm{~min}$ post-protocol (figure 4 ).

\section{LESSONS AND LIMITATIONS}

The protocol was used to determine necessary resources needed to provide excellent care for patients with SMPE. The project led to a standardised institutional SMPE definition and treatment pathway. Stat echocardiogram is now performed on patients with SMPE in addition to reporting RV/LV ratios. The protocol improved emergency medicine and critical care physician comfort and knowledge of appropriate ultrasound-enhanced thrombolysis treatment without an increase or decrease in utilisation.

Our SMPE protocol was adapted from the PERT reported by Kabrhel et al with several key differences. According to their protocol, the PERT was activated by a referring physician via a 24 hour phone line for patients with radiographically confirmed $\mathrm{PE}$ or for patients with high clinical suspicion of $\mathrm{PE}$, including patients with low risk, submassive and massive PE. The PERT examined clinical information, communicated via a multidisciplinary online meeting and sent back a recommendation to the referring physician. ${ }^{56}$ At our institution, activation of the protocol was specific to patients with SMPE and initiated a computerised order set that included pre-checked echocardiogram, ancillary studies and official consultations with critical care and interventional cardiology. As part of the official consultation, emergency physicians paged critical care and interventional cardiologists who then came to the $\mathrm{ED}$ for an in-person consultation. In addition, the referring physician was involved in the treatment decision-making process.

\section{B) \% Patients with Cardiologist Consultation}
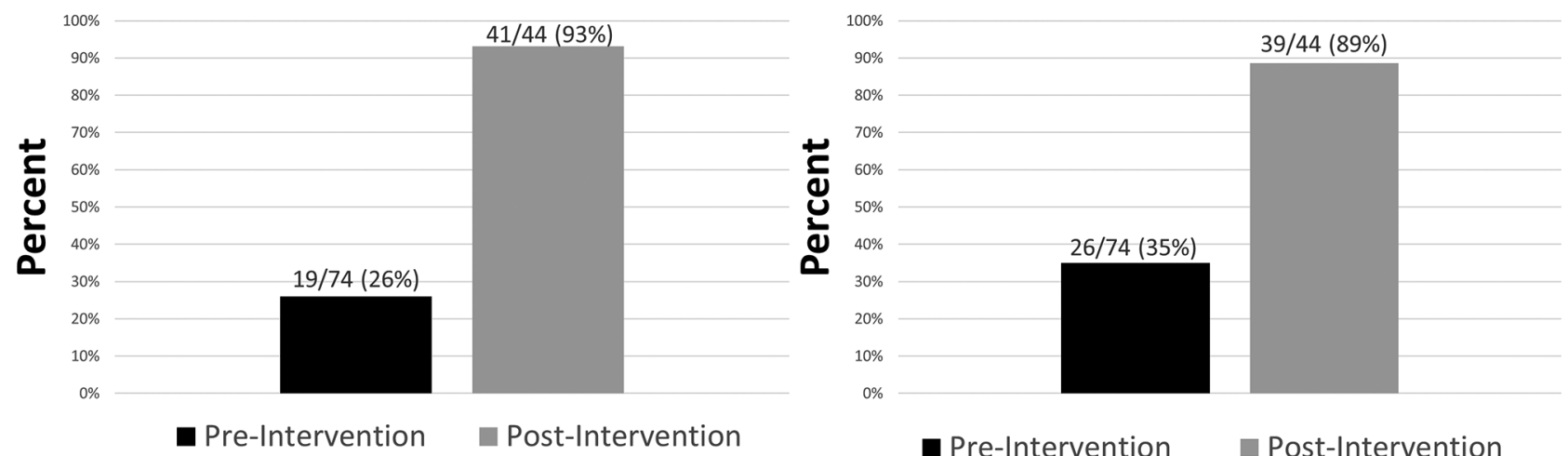

Figure 2 The percent of patients with submassive pulmonary embolus with critical care $(A)$ and cardiology consultations (B) was significantly increased post-protocol implementation, two-proportion test, $p<0.001$. 


\section{Patient Arrival to Echocardiogram}

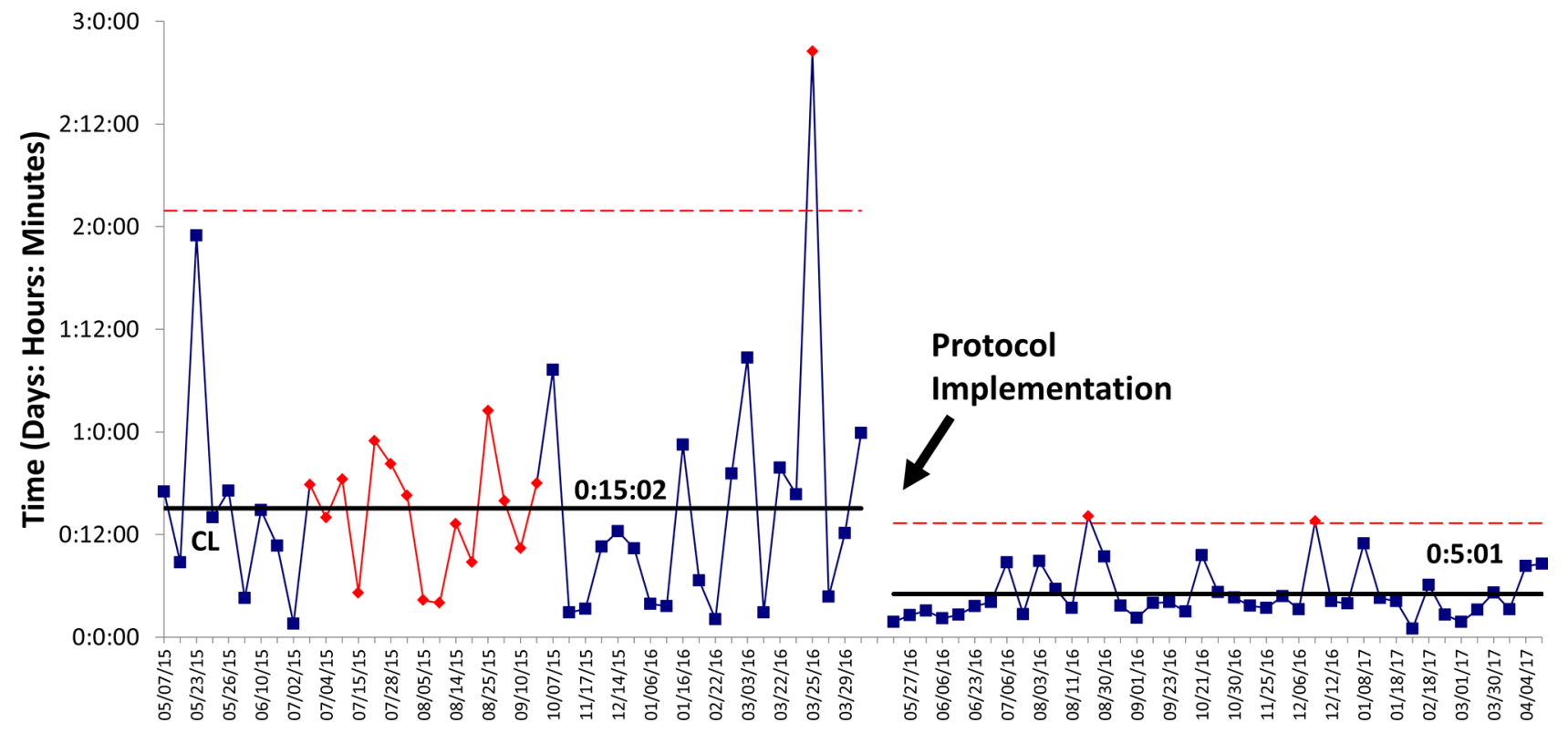

Figure 3 Patient arrival to echocardiogram was significantly reduced post-protocol implementation. The upper control limit, shown as the dotted line, represents three standard deviations from the mean. The solid centre line represents the average.

A limitation of this study was that it was performed at a single institution and results cannot be generalised. Additionally, cardiology was the only service performing ultrasound-enhanced thrombolysis; therefore, this protocol may not be applicable to all institutions. We learnt that even when providing education on the protocol several times, utilising several communication methods, not everyone seemed aware of the protocol and failed to follow the protocol. A limitation of the study was that knowledge of SMPE recognition, therapy and the protocol was not directly measured. RV/LV ratios were not documented for all acute patients with PE resulting in fewer total patients in the post-intervention group. Continued communication efforts are essential even after the initial education.

Prior to the protocol, multiple therapeutic pathways existed. The standardised approach of the protocol provided clear direction and was well received by emergency medicine physicians. The goal of the standardised protocol to reduce variability in SMPE recognition and treatment was met. After 1 year, the protocol was changed so that a stat echocardiogram was no longer required for all patients but instead is at the discretion of the emergency physician and critical care physicians. Therefore,

\section{Patient Arrival to Anticoagulation}

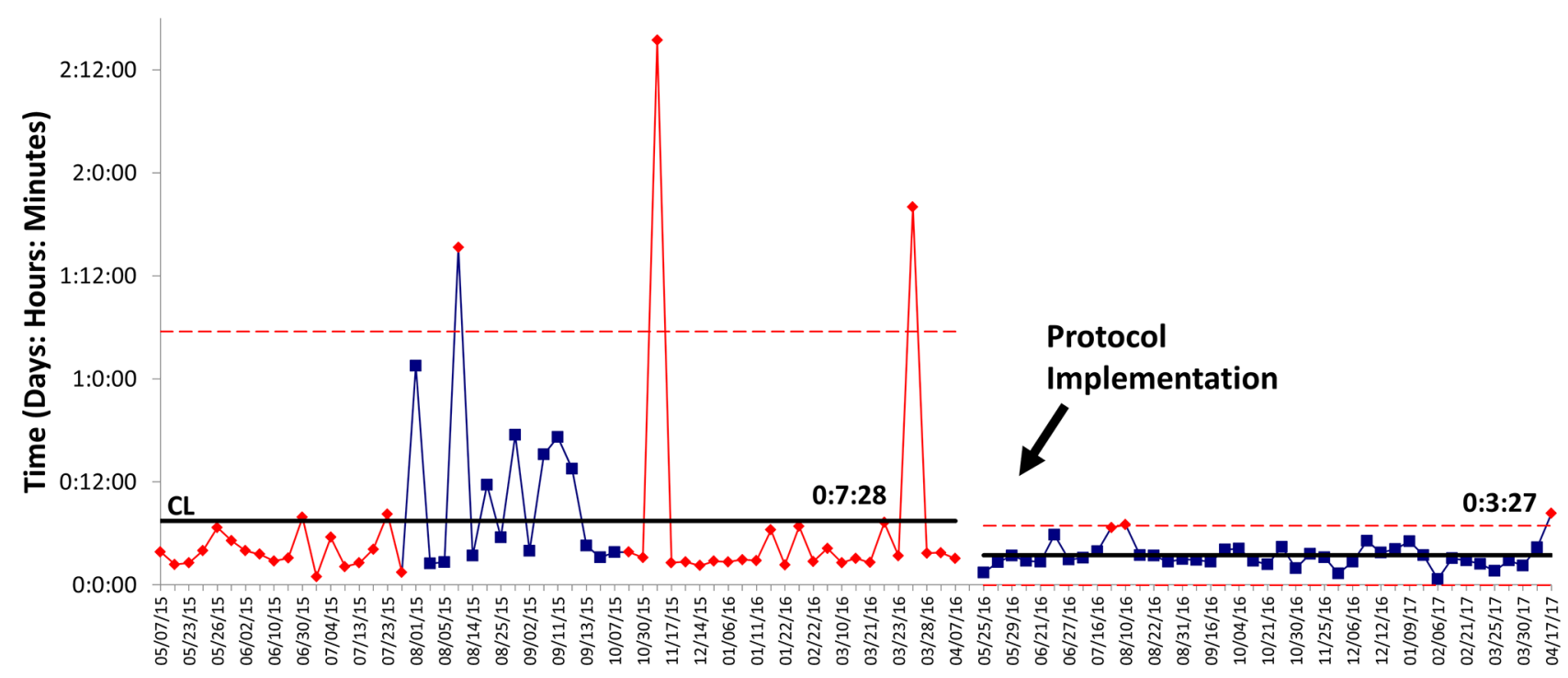

Figure 4 Patient arrival to anticoagulation was reduced post-protocol implementation. 
sustainability of the protocol was not tested. Of note, the volume of stat echocardiograms has not changed since completion of the project. Protocols can be used as an interim approach to standardised care, teach physicians an institutionally preferred process and increase comfort in treating diagnoses which may have variability in presentation, risk stratification methods and treatment pathways.

\section{CONCLUSION}

The SMPE protocol has effectively standardised the definition and treatment approach to patients with SMPE. The percent of patients with SMPE with critical care and cardiology consultations was increased significantly. Patient arrival to echocardiogram was significantly reduced in addition to reduced patient arrival to anticoagulation. The standardised approach successfully clarified the diagnosis and treatment of patients with SMPE and encouraged collaboration between specialties.

Contributors KG contributed to study concept and design, acquisition of data, analysis and interpretation of data and drafting and critical revision of the manuscript. CJ contributed to acquisition of data, drafting and critical revision of the manuscript and statistical expertise. KC contributed to acquisition, analysis and interpretation of the data. PW contributed to study concept and design and study supervision. DG contributed to study concept and design, analysis and interpretation of data, drafting and critical revision of the manuscript and study supervision. All contributors reviewed the manuscript.
Funding The authors have not declared a specific grant for this research from any funding agency in the public, commercial or not-for-profit sectors.

Competing interests None declared.

Patient consent Not required.

Provenance and peer review Not commissioned; externally peer reviewed.

Open access This is an open access article distributed in accordance with the Creative Commons Attribution Non Commercial (CC BY-NC 4.0) license, which permits others to distribute, remix, adapt, build upon this work non-commercially, and license their derivative works on different terms, provided the original work is properly cited, appropriate credit is given, any changes made indicated, and the use is non-commercial. See: http://creativecommons.org/licenses/by-nc/4.0/.

\section{REFERENCES}

1. Horlander KT, Mannino DM, Leeper KV. Pulmonary embolism mortality in the United States, 1979-1998: an analysis using multiple-cause mortality data. Arch Intern Med 2003;163:1711-7.

2. Jaff MR, McMurtry MS, Archer SL, et al. Management of massive and submassive pulmonary embolism, iliofemoral deep vein thrombosis, and chronic thromboembolic pulmonary hypertension: a scientific statement from the American Heart Association. Circulation 2011;123:1788-830.

3. Kearon C, Akl EA, Comerota AJ, et al. Antithrombotic therapy for VTE disease: antithrombotic therapy and prevention of thrombosis, 9th ed: American College of chest physicians evidence-based clinical practice guidelines. Chest 2012;141:e419S-96.

4. Konstantinides S, Torbicki A, Agnelli G, et al. ESC guidelines on the diagnosis and management of acute pulmonary embolism. Eur Heart $J$ 2014;2015:3033-69.

5. Kabrhel C, Jaff MR, Channick RN, et al. A multidisciplinary pulmonary embolism response team. Chest 2013;144:1738-9.

6. Kabrhel C, Rosovsky R, Channick R, et al. A multidisciplinary pulmonary embolism response team: initial 30-month experience with a novel approach to delivery of care to patients with submassive and massive pulmonary embolism. Chest 2016;150:384-93. 